\title{
CONSIDERACIÓN DE LAS CONSECUENCIAS FUTURAS: PROPIEDADES PSICOMÉTRICAS, DISTRIBUCIÓN GEOGRÁFICA Y ASOCIACIÓN CON EL CONSUMO DE SUSTANCIAS EN UNA MUESTRA REPRESENTATIVA DE MONTEVIDEO, URUGUAY ${ }^{\text {¿ }}$
}

\author{
Lucía Stephanie Alvarez-Nuñez ${ }^{1} \& 2 \oplus$, Hugo Selma ${ }^{3}$, \& Alejandro \\ Vásquez-Echeverría ${ }^{2}$
}

Universidad de la República, Montevideo, Uruguay.

\begin{abstract}
RESUMEN
La escala de Consideración de las Consecuencias Futuras (CCF) evalúa el grado que las personas son influenciadas por las consecuencias inmediatas o distantes de sus comportamientos. En una muestra representativa de Montevideo, este estudio procuró explorar sus propiedades psicométricas, establecer sus baremos poblacionales, explorar su distribución territorial y evaluar las asociaciones de la CCF con el consumo de sustancias psico-activas. En este estudio, la solución de dos factores provee mejor ajuste, con adecuada confiabilidad. Las CCF presentan diferencias significativas según edad y sexo por lo que se ofrecen baremos específicos. Asimismo, encontramos evidencia mixta sobre su distribución territorial y un efecto pequeño sobre el consumo de algunas sustancias psicoactivas. Se discuten los resultados en función del rol de la CCF para explicar el comportamiento con consecuencias intertemporales.
\end{abstract}

Palabras Claves

consideración de las consecuencias futuras; propiedades psicométricas; baremo; estatus socioeconómico; consumo de sustancias

\section{AbSTRaCT}

The Consideration of Future Consequences (CFC) scale assesses the degree to which people are influenced by the immediate or distant consequences of their behaviors. In a representative sample of Montevideo, the aim of this study is to explore its psychometric properties, establish its population scales, explore its territorial distribution and evaluate the associations of the CFC with the consumption of drugs. In this study, the two-factor solution provides better fit, with adequate reliability. The CFC present significant differences according to age and sex, for which specific scales are offered. Likewise, we found mixed evidence on their territorial distribution and a small effect size on the consumption of some substances. Results are discussed based on the role of the CFC to explain behavior with intertemporal consequences.

\section{Keywords}

consideration of future consequences; psychometric properties; scale scores, socioeconomic status; substance use

\footnotetext{
${ }^{1}$ Correspondence about this article should be addressed to Lucía Stephanie Alvarez-Nuñez: lucia.alvarez@psico.edu.uy

${ }^{2}$ Instituto de Fundamentos y Métodos en Psicología. Facultad de Psicología, Universidad de la República. Tristán Narvaja 1676, Montevideo, Uruguay.

${ }^{3}$ Instituto de Psicología Clínica de Facultad de Psicología, Facultad de Psicología, Universidad de la República. Tristán Narvaja 1676, Montevideo, Uruguay.
} 


\section{THE CONSIDERATION OF FUTURE CONSEQUENCES: PSYCHOMETRIC PROPERTIES, GEOGRAPHICAL DISTRIBUTION AND ASSOCIATION WITH SUBSTANCE USE IN A REPRESENTATIVE SAMPLE OF MONTEVIDEO, URUGUAY}

\section{Introducción}

La Consideración de las Consecuencias Futuras (en adelante CCF) es un rasgo de personalidad mediante la cual se puede conocer en qué forma las personas sopesan y son influenciadas por las consecuencias inmediatas y las distantes de sus acciones para tomar decisiones. A través de este constructo, se puede explicar cómo las personas resuelven los dilemas temporales de su diario vivir. Por ejemplo, las personas deben sopesar los resultados de consumir sustancias psicoactivas (patrón conductual asociado a beneficios inmediatos placenteros, pero con consecuencias distantes negativas para su salud) versus tener hábitos de vida saludables, como comer saludablemente o realizar actividad física diariamente (acciones con resultados distantes asociados a mayor esperanza de vida y salud general).

La CCF es evaluada mediante la Escala Consideración de las Consecuencias Futuras (ECCF). Este instrumento fue propuesto por Strathman, Gleicher, Boninger, \& Edwards (1994) conformado por 12 ítems y una estructura unifactorial. Más recientemente, Joireman, Balliet, Sprott, Spangenberg, \& Schultz (2008) propusieron que la ECCF se compone de dos subescalas, una de consideración de las consecuencias inmediatas (CCF-I) y otra de consideración de las consecuencias futuras (CCF-F). En esta línea, Joireman, Shaffer, Balliet, \& Strathman (2012) propusieron la ampliación de la escala de 12 a 14 ítems, agregando dos en la subescala CCF-F con el objetivo de mejorar su confiabilidad. Las investigaciones subsiguientes sobre la dimensionalidad del constructo apoyan de forma empírica y mayoritaria la solución de dos factores correlacionados (Camus, Berjot, \& Ernst-Vintila, 2014; Joireman et al., 2008, 2012; Nigro, Cosenza, Ciccarelli, \& Joireman, 2016; Toepoel, 2010; Vásquez-Echeverría, Antino, Alvarez-Nuñez, \& Rodríguez-Muñoz, 2018) aunque otras soluciones factoriales fueron propuestas (por ejemplo: Zhang, Kong, Zhang, \& Li, 2015).

La ECCF-14 ha sido traducida y validada a diferentes idiomas y culturas. Por ejemplo: en francés (Camus et al., 2014), inglés (McKay, Perry, Percy, \& Cole, 2016), italiano (Nigro et al., 2016) y en español (Vásquez-Echeverría et al., 2018). Para el caso del castellano, se han reportado adecuados valores de confiabilidad y validez de la escala CCF de 14 ítems con una estructura de dos factores correlacionados (Vásquez-Echeverría 
et al., 2018). Todos los estudios mencionados previamente se han realizado con muestras por conveniencia y en su mayoría, con estudiantes universitarios. Hasta ahora no se ha evaluado las propiedades psicométricas de la ECCF-14 con una muestra representativa de regiones o países, a nivel hispanoamericano, ni a nivel mundial.

El nivel de CCF de las personas ha sido asociado con diversas conductas relevantes para el bienestar. En una revisión, Joireman, Strathman, \& Balliet (2006) plantearon cuatro dominios de comportamiento relacionados con la CCF: (a) Comportamientos de salud, Conductas de Riesgo y Logros Académicos (e.g., Beenstock, Adams, \& White, 2011), (b) Agresión (e.g., Joireman, Becker, Barbosa-Leiker, \& Duell, 2005); (c) Comportamiento Organizacional Prosocial (e.g., Balliet \& Ferris, 2013) y (d) Actitudes y conductas proambientales (e.g., Lindsay \& Strathman, 1997). En relación al primer dominio, los comportamientos relacionados al cuidado de la salud implican costos inmediatos en términos de tiempo, dinero y diversas gratificaciones dejadas de lado para lograr beneficios a largo plazo (Adams, 2012). Es así que la forma en que un individuo considere y anticipe los resultados futuros incide en los comportamientos saludables que realice. En relación a este punto, se ha comprobado que mayor orientación al futuro se asocia a menor frecuencia de consumo de sustancias psicoactivas, consumo de tabaco y bebidas alcohólicas (Adams, 2012; Daugherty \& Brase, 2010). A su vez, se ha evidenciado el poder predictivo de la CCF sobre el consumo de sustancias psicoactivas (e.g.: McKay et al., 2016; Percy, McKay, Perry, \& Cole, 2018).

\section{Este estudio}

Utilizando datos de una muestra representativa de Montevideo (Uruguay) este trabajo tiene cuatro objetivos. El primero es evaluar la estructura factorial y la confiabilidad de la ECCF-14. Hasta ahora, no se conocen estudios que reporten estas propiedades psicométricas utilizando muestras representativas, ni en su versión en español, ni a nivel internacional. En base a la literatura previa, esperamos confirmar la solución de dos factores correlacionados y niveles de confiabilidad superiores a .70.

Un segundo objetivo es establecer los baremos poblacionales de la Escala CCF. Contar con la muestra representativa nos permite asegurar que las puntuaciones obtenidas en este estudio sirvan de orientación para que otros investigadores que trabajan con poblaciones específicas puedan cotejar sus resultados. Hasta nuestro conocimiento, no existen baremos disponibles para ninguna de las versiones de la Escala CCF. 
Tercero, pretendemos explorar la distribución territorial de las puntuaciones de la Escala CCF en Montevideo, los cuales presentan marcadas diferencias socioeconómicas. Varios académicos han señalado que la pobreza se relaciona con una mayor orientación hacia el presente (Adams \& White, 2009; Haushofer \& Fehr, 2014; Pepper \& Nettle, 2017; Vásquez-Echeverría, Tomás, \& Cruz, 2019) debido a que la privación de recursos y menor control del ambiente hace que las conductas que tienen consecuencias a largo plazo sean más inciertas en cuanto a su utilidad y retorno. Este fenómeno se ha postulado que es ambiental (o sea, un proceso colectivo), ya que factores como la tasa de criminalidad, la expectativa de vida y/o las tasas de desempleo a nivel de los barrios permean las forma en que cada persona valora el futuro (Pepper \& Nettle, 2017). Para comprobar este fenómeno en una muestra representativa, analizaremos el desempeño en las puntuaciones de CCF en los siete grupos zonales de Montevideo.

Cómo último objetivo nos propusimos explorar las asociaciones entre las puntuaciones de CCF y la frecuencia de consumo de sustancias psicoactivas. Basados en la literatura previa (Daugherty \& Brase, 2010; McKay et al., 2016; Percy et al., 2018) esperamos que a mayor consideración de las consecuencias distantes y menor consideración de las consecuencias inmediatas sean factores protectores relativos al consumo de sustancias psicoactivas a nivel poblacional.

\section{Método}

\section{Participantes y procedimiento}

Participaron 1.319 personas de entre 18 y 94 años $(M=37.77$; DT=16.16), de los cuales 726 son mujeres $(\mathrm{M}=37.88$; $\mathrm{DT}=16.32)$ y 593 hombres $(\mathrm{M}=37.64$; $\mathrm{DT}=15.98)$.

Para alcanzar la representatividad de la muestra se sortearon 438 secciones censales del departamento de Montevideo. Dentro de cada sección censal se encuestaron a cuatro participantes. Dentro de cada sección censal sorteada, se seleccionaba al azar una esquina y desde allí se comenzaba a visitar los domicilios, invitando a un solo residente por domicilio a participar del estudio. Al alcanzar la cuarta aceptación dentro de una sección censal, se pasaba a la siguiente. Adicionalmente, los encuestadores, debían cumplir con cuotas según sexo y edad basadas en los datos del censo de población de 2011 del Instituto Nacional de Estadística (Instituto Nacional de Estadística, 2011). Las secciones censales fueron clasificadas en zonas censales según los criterios de Ponce \& Tubbio (2013) de valor inmobiliario de los barrios, para analizar la distribución territorial 
de las puntuaciones de CCF. Debido a que en dicha clasificación no se encuentran todos los barrios que, sí incluye nuestro estudio, dicho análisis está restringido a una muestra más pequeña. Dicha agrupación se compone de siete grupos que describimos a continuación. Grupo 1: Colón, Maroñas, Peñarol; Grupo 2: Malvín Norte, Villa Española, Goes, Sayago; Grupo 3: Ciudad Vieja, Aguada, La Comercial, Reducto y Brazo Oriental; Grupo 4: Cordón, Centro, Prado, Tres Cruces, La Blanqueada; Grupo 5: Buceo, Parque Batlle y Parque Rodó; Grupo 6: Pocitos y Malvín; Grupo 7: Carrasco y Punta Carretas.

En la puerta del hogar, el encuestador explicaba los objetivos del estudio. Si la persona aceptaba formar parte, como primer paso se le solicitaba la firma de un consentimiento informado aprobado por el Comité de Ética de la Universidad implicada y en acuerdo con la legislación vigente. Una vez firmado, como segundo paso, se preguntaba oralmente el cuestionario sociodemográfico ad-hoc. Como tercer paso, se entregaba la ECCF (y otro cuestionario no analizado en este estudio) para que el participante autocompletara. Para mayor tranquilidad del participante en la cumplimentación, el entrevistador coordinaba un horario para pasar a retirarlos mientras visitaba otros hogares.

\section{Instrumentos}

Cuestionario sociodemográfico Ad-hoc. Se recolectó información sobre el sexo, edad y nivel educativo alcanzado por cada participante. El nivel educativo tiene las siguientes opciones de respuestas: $0=$ No posee primaria; $1=$ Primaria incompleta; $2=$ Primaria completa; $3=$ secundaria incompleta $; 4=$ secundaria completa $; 5=$ enseñanza técnica UTU o similar incompleto; 6= enseñanza técnica UTU o similar completo; 7= Estudios terciarios/universidad/magisterio incompleto; $8=$ Estudios terciarios/universidad/magisterio completo; $9=$ Posgrado incompleto; $10=$ Posgrado completo.

Cuestionario de consumo de sustancias Ad-hoc. Se preguntó el tipo de sustancias consumidas, dentro de las siguientes opciones: cerveza, vino, tragos destilados, tabaco y marihuana. Para cada sustancia las personas debían responder cuál era la frecuencia de consumo, utilizando la siguiente escala: $0=$ Nunca, $1=$ Algunas veces; $2=$ Fines de semana; 3= Todos los días. Para las bebidas alcohólicas se realizó una sumatoria de las opciones brindadas (cerveza, vino y tragos destilados). 
Escala de Consideración de las Consecuencias Futuras de 14 ítems (ECCF-14). En este estudio se empleó la versión de la ECCF adaptada al español por VásquezEcheverría et al. (2018). La ECCF-14 está compuesta por 14 ítems, evaluados con una escala Likert con siete valoraciones $(1=$ Nada característico; $7=$ Totalmente característico). El instrumento se compone de dos dominios: CCF-I y CCF-F. En Vásquez-Echeverría et al. (2018) los omega de McDonald son de .74 para CCF-I y de .78 para CCF-F. Asimismo, se reporta muy buenos índices de ajuste confirmatorio para la solución de dos factores correlacionados, análisis para el cual se correlacionan el término de error del ítem 3 con el 4 y del ítem 13 con el 14 (Joireman et al., 2012; Murphy, Cadogan, \& Dockray, 2019; Vásquez-Echeverría et al., 2018). Debido a un error técnico, en esta encuesta se utilizó una redacción anterior del ítem 5 a la utilizada en dicha validación.

\section{Análisis de datos}

Para los análisis se utilizó el programa SPSS 20 y MPLUS 8.0. El primer programa fue empleado para realizar los estadísticos descriptivos, estimar los coeficientes de correlación, realizar el baremo (para este análisis se seleccionaron los puntos de corte correspondientes a los percentiles: $5 ; 10 ; 25 ; 33.3 ; 50 ; 66.6 ; 75 ; 90$ y 95), comparación de medias (mediante una prueba t y ANOVA mediante el post hoc Tukey) y análisis de regresión lineal múltiple. El análisis de regresión lineal múltiple fue realizado por pasos. En el primer paso, se incluyó la edad, sexo y máximo nivel educativo como variables control. En el segundo paso, se incluyó CCF-I y CCF-F. En este análisis no se incluye a puntuación total de la ECCF (en adelante CCF-T) por la alta correlación que presenta con las subescalas del instrumento. El segundo programa fue empleado para evaluar la estructura interna mediante un análisis factorial confirmatorio (AFC) y confiabilidad de la escala. Para estos análisis se seleccionó el estimador MLR (del inglés Maximum Likelihood Robust), con el fin de calcular las estimaciones de máxima verosimilitud con errores estándares robustos (Byrne, 2013).

En el AFC se testeó el modelo unifactorial de Strathman et al. (1994), dos factores correlacionados de Joireman et al. (2012) y modelo bifactorial de McKay et al. (2016). Luego, se evaluó la consistencia interna mediante el omega de McDonald, los valores $\geq$ .70 fueron considerados adecuados (Cicchetti, 1994; Viladrich, Angulo-Brunet, \& Doval, 2017), entre .80 a .89 buenos $y \geq .90$ excelentes (Cicchetti, 1994). 
Tratamiento de la base de datos

Se excluyeron las respuestas de 39 participantes por contestar más del $80 \%$ el mismo valor en algún ítem de la ECCF y 51 por ser identificados como valores extremos multivariados a través del cálculo de la distancia de Mahalanobis.

\section{Resultados}

\section{Estadísticos descriptivos y correlaciones entre las variables del estudio}

En la Tabla 1 se presentan los estadísticos descriptivos y coeficientes de correlaciones inter-ítem de la ECCF. Se utilizó todo el rango de respuesta de la escala en todos los ítems. No se observan distorsiones graves en la curtosis y simetría. El ítem 5 es la variable con mayor cantidad de correlaciones sin significancia estadística. A su vez, se observan asociaciones sin significancia estadística en el ítem 12 y 14.

\section{Tabla 1}

Estadísticos descriptivos de los ítems de la escala CCF-14 y correlaciones inter-ítem

\begin{tabular}{|c|c|c|c|c|c|c|c|c|c|c|c|c|c|c|c|c|}
\hline Ítems & $\mathrm{M}$ (DT) & As & $\mathrm{Cu}$ & 2 & 3 & 4 & 5 & 6 & 7 & 8 & 9 & 10 & 11 & 12 & 13 & 14 \\
\hline Ítem 1 & $4.92(1.87)$ & -0.69 & -0.46 & $.38^{* * *}$ & $-.26^{* *}$ & $-.16^{* *}$ & .04 & $.18^{* *}$ & $.28^{* *}$ & $.32^{* *}$ & $-.16^{* *}$ & $-.23^{* *}$ & $-.21^{* *}$ & $-.08^{* *}$ & $.37^{* *}$ & $.37^{* * *}$ \\
\hline Ítem 2 & $3.68(2.04)$ & 0.14 & -1.20 & & $-.11^{* *}$ & $-.08^{* *}$ & -.01 & $.25^{* *}$ & $.26^{* *}$ & $.26^{* *}$ & $-.06^{*}$ & $-.07^{*}$ & $-.17^{* *}$ & -.04 & $.21^{* *}$ & $.23^{* *}$ \\
\hline Ítem 3 & $2.86(2.01)$ & 0.79 & -0.68 & & & $.54^{* *}$ & $.14^{* *}$ & $-.07^{*}$ & $-.15^{* *}$ & $-.22^{* *}$ & $.27^{* * *}$ & $.31^{* *}$ & $.48^{* *}$ & $.34^{* *}$ & $-.25^{* *}$ & $-.21^{* *}$ \\
\hline Ítem 4 & $2.83(1.97)$ & 0.81 & -0.60 & & & & $.17^{* * *}$ & $-.07^{*}$ & $-.09^{* *}$ & $-.19^{* *}$ & $.27^{* *}$ & $.32^{* *}$ & $.47^{* *}$ & $.33^{* * *}$ & $-.22^{* *}$ & $-.21^{* *}$ \\
\hline Ítem 5 & $4.59(2.01)$ & -0.44 & -0.97 & & & & & $-.07^{*}$ & .01 & .02 & $.12^{* *}$ & $.14^{* *}$ & $.19^{* *}$ & $.14^{* * *}$ & $.09^{* * *}$ & .05 \\
\hline Ítem 6 & $3.72(2.10)$ & 0.08 & -1.33 & & & & & & $.21^{* *}$ & $.24^{* *}$ & $-.07^{* *}$ & $-.17^{* *}$ & $-.16^{* *}$ & $-.12^{* *}$ & $.19^{* *}$ & $.25^{* *}$ \\
\hline Ítem 7 & $4.65(1.92)$ & -0.45 & -0.87 & & & & & & & $.39^{* *}$ & $-.21^{* *}$ & $-.10^{* *}$ & $-.09^{* *}$ & .00 & $.31^{* *}$ & $.29^{* *}$ \\
\hline Ítem 8 & $4.88(1.83)$ & -0.57 & -0.62 & & & & & & & & $-.10^{* * *}$ & $-.18^{* *}$ & $-.14^{* *}$ & $-.09^{* * *}$ & $.34^{* *}$ & $.36^{* *}$ \\
\hline Ítem 9 & $3.29(2.01)$ & 0.48 & -1.02 & & & & & & & & & $.45^{* *}$ & $.43^{* *}$ & $.30^{* * *}$ & $-.11^{* *}$ & $-.13^{* * *}$ \\
\hline Ítem 10 & $2.80(1.91)$ & 0.82 & -0.44 & & & & & & & & & & $.45^{* *}$ & $.31^{* *}$ & $-.17^{* *}$ & $-.16^{* *}$ \\
\hline Ítem 11 & $3.24(2.06)$ & 0.52 & -1.03 & & & & & & & & & & & $.44^{* * *}$ & $-.16^{* *}$ & $-.19^{* *}$ \\
\hline Ítem 12 & $3.61(1.94)$ & 0.29 & -0.96 & & & & & & & & & & & & -.05 & $-.09^{* *}$ \\
\hline Ítem 13 & $5.28(1.81)$ & -0.99 & 0.04 & & & & & & & & & & & & & $.65^{* *}$ \\
\hline Ítem 14 & $4.66(1.97)$ & -0.52 & -0.88 & & & & & & & & & & & & & \\
\hline
\end{tabular}

Notas: $\mathrm{M}(\mathrm{DT})=$ Media (Desviación Típica); AS= Asimetría; $\mathrm{Cu}=$ Curtosis.

\section{Consistencia interna y estructura factorial de la ECCF.}

La CCF-T presenta un omega de McDonald de .73, CCF-I de .69 y CCF-F de .67. Si el ítem 5 se elimina la confiabilidad en CCF-T aumenta a .74 y CCF-I a .70. La media de correlación inter-ítem de CCF-I es de .31. El ítem 5 es el que presenta menor 
correlación, la media de correlación inter-ítem aumenta .07 si el mismo es eliminado. La media de correlación inter-ítem de CCF-F es de .30 .

Dado los inconvenientes psicométricos del ítem 5 reportados en una versión anterior de la ECCF (Vásquez Echeverría, Martín, Ortuño, Esteves, \& Joireman, 2017) los AFC se realizaron tanto con el ítem 5 y sin el mismo. Al comparar los resultados de ambos análisis se evidencia que los índices de ajustes y cargas factoriales de los modelos mejoran al excluir el ítem 5. Dado que hay evidencia para su exclusión en este estudio, en los análisis siguientes se presentan los resultados de los modelos sin dicho ítem.

CCF-T presenta una media de 4.71 (DT=1.05), CCF-I de 3.11 (DT=1.38) y CCFF de 4.54 (DT=1.22). En la Tabla 2 se presentan los índices de ajuste de cada modelo evaluado en el AFC. El modelo unifactorial presenta índices de ajuste subóptimos para esta muestra. A su vez, el modelo bifactor presenta CFI adecuado, pero TLI y RMSEA subóptimos. El modelo de dos factores correlacionados presentar índices de ajustes adecuado para la muestra en todas las propiedades evaluadas.

\begin{tabular}{lcccccc}
\hline Tabla 2 & \multicolumn{1}{l}{$l$} \\
Índices de ajustes de los modelos factoriales testeados para la ECCF & \\
\hline Modelo & $\chi 2$ & Gl & CFI & TLI & RMSEA (90\% CI) & SRMR \\
\hline I. Unifactorial & $876.664^{*}$ & 63 & 0.734 & 0.671 & $0.099(0.093-0.105)$ & 0.093 \\
II. Dos factores correlacionados & $284.966^{*}$ & 62 & 0.927 & 0.908 & $0.052(0.046-0.058)$ & 0.042 \\
III. Bifactor & $321.438^{*}$ & 52 & 0.912 & 0.868 & $0.063(0.056-0.069)$ & 0.038 \\
\hline
\end{tabular}

En la Tabla 3 se presentan las cargas factoriales del modelo de dos factores correlacionados y modelo bifactorial. En el primer modelo todas las saturaciones son mayores a .40 (con la excepción del ítem 6 que presenta un $\beta$ de .39), positivas y estadísticamente significativas. La puntuación factorial de las subescalas presenta una correlación negativa $(\mathrm{r}=-.40, \mathrm{p}<.001)$. En el modelo bifactorial, el factor general presenta saturaciones estadísticamente significativas, pero con saturaciones menores a .30. En el factor específico referido a CCF-I se observan cargas mayores a .40, estadísticamente significativas y en la valencia esperada. En CCF-F todas las cargas son $<.40$ (con la excepción del ítem 13). Además, cuatro saturaciones no son significativas. 
Tabla 3

Cargas factoriales de los dos modelos testeados

\begin{tabular}{|c|c|c|c|c|c|}
\hline \multirow[b]{3}{*}{ Ítem } & \multicolumn{2}{|c|}{ Modelo de dos factores } & \multicolumn{3}{|c|}{ Modelo bifactorial } \\
\hline & CCF-I & CCF-F & Factor General & CCF-I & CCF-F \\
\hline & $\beta$ & $\beta$ & $\beta$ & $\beta$ & $\beta$ \\
\hline 1 & & 0.61 & 0.59 & & $-0.13^{(1)}$ \\
\hline 2 & & 0.49 & 0.47 & & -0.28 \\
\hline 3 & 0.59 & & -0.33 & 0.56 & \\
\hline 4 & 0.57 & & -0.28 & 0.57 & \\
\hline 6 & & 0.39 & 0.37 & & $-0.14^{(1)}$ \\
\hline 7 & & 0.53 & 0.51 & & $-0.15^{(1)}$ \\
\hline 8 & & 0.60 & 0.57 & & $-0.13^{(1)}$ \\
\hline 9 & 0.56 & & -0.20 & 0.50 & \\
\hline 10 & 0.60 & & -0.26 & 0.52 & \\
\hline 11 & 0.78 & & -0.27 & 0.72 & \\
\hline 12 & 0.55 & & -0.11 & 0.56 & \\
\hline 13 & & 0.56 & 0.72 & & 0.47 \\
\hline 14 & & 0.58 & 0.70 & & 0.32 \\
\hline
\end{tabular}

\section{Puntajes de referencia a la norma}

Para proceder a establecer los puntajes de referencia a la norma de la ECCF en Montevideo, en primer lugar, analizamos posibles diferencias significativas según sexo y edad. Para esto, se realizó una comparación de medias según sexo que mostró diferencias estadísticamente significativas únicamente para CCF-I. Sobre este punto, se observa que, en promedio, los hombres reportan mayor CCF-I $(\mathrm{M}=3.19$; $\mathrm{DT}=1.38)$ que las mujeres $(\mathrm{M}=3.04 ; \mathrm{DT}=1.38)$. La diferencia es estadísticamente significativa t $(1317)=2.08, \mathrm{p}$ $<.05)$.

Adicionalmente, realizamos un ANOVA por edad, para lo cual se establecieron cuatro grupos de edad (adultez joven de 18 a 29 años; adultez media 30 a 49, adultez tardía 50 a 65 y adultos mayores 66 o más). Las diferencias de media por tramo de edad son significativas únicamente en CCF-I: $F(3,1314)=7.71, p<.001$. Las diferencias, según la comparación post-hoc de Tukey son significativas entre adultos mayores $(\mathrm{M}=3.65 ; \mathrm{DT}=1.53)$ con: adultos jóvenes $(\mathrm{M}=3.02 ; \mathrm{DT}=1.35)$ y adultos medios $(\mathrm{M}=$ 3.03; DT=1.36).

Por lo tanto, se establecieron los baremos para CCF-T, CCF-I y CCF-F en función de la población total. A su vez, se establecieron los baremos en base a criterios de edad y 
sexo para CCF-I. Los percentiles que corresponden a cada puntuación bruta pueden consultarse en el Anexo 1.

\section{Distribución territorial de las puntuaciones de CCF}

En la Tabla 4 pueden verse las medias en las puntuaciones de CCF y nivel educativo según grupo zonal (ordenados de menor a mayor según el valor medio de los inmuebles de acuerdo a la clasificación de Ponce \& Tubbio, 2013). De forma general, se observa que los grupos zonales de mayor opulencia, presentan puntajes más bajos en CCF-I, más altos en CCF-F y mayor nivel educativo. Por el contrario, los grupos zonales 1 y 2 (los de menor valor inmobiliario) son los que presentan mayor CCF-I y menor CCFF, así como menor nivel educativo. El cambio de tendencia se da a partir del grupo zonal 3.

\begin{tabular}{|c|c|c|c|c|c|c|c|}
\hline & $\begin{array}{l}\text { las cons } \\
\text { alor inmo }\end{array}$ & $\begin{array}{l}\text { lencias } \\
\text { liario) }\end{array}$ & ras y ni & educativ & egún gru & zonal ( & lados de \\
\hline & \multicolumn{7}{|c|}{ Grupo zonal } \\
\hline & $\begin{array}{c}1 \\
(n=62)\end{array}$ & $\begin{array}{c}2 \\
(\mathrm{n}=85)\end{array}$ & $\begin{array}{c}3 \\
(\mathrm{n}=54)\end{array}$ & $\begin{array}{c}4 \\
(\mathrm{n}=200)\end{array}$ & $\begin{array}{c}5 \\
(\mathrm{n}=130)\end{array}$ & $\begin{array}{c}6 \\
(\mathrm{n}=228)\end{array}$ & $\begin{array}{c}7 \\
(n=50)\end{array}$ \\
\hline \multirow{2}{*}{ CCF-I } & 3.22 & 3.19 & 2.85 & 3.15 & 2.90 & 2.85 & 2.84 \\
\hline & $(1.40)$ & $(1.30)$ & $(1.40)$ & (1.34) & $(1.29)$ & $(1.25)$ & (1.41) \\
\hline \multirow{2}{*}{ CCF-F } & 4.32 & 4.25 & 4.85 & 4.51 & 4.74 & 4.61 & 4.80 \\
\hline & (1.18) & (1.19) & (1.19) & (1.29) & $(0.98)$ & (1.12) & (1.11) \\
\hline \multirow{2}{*}{ CCF-T } & 4.53 & 4.51 & 4.99 & 4.67 & 4.91 & 4.86 & 4.97 \\
\hline & $(1.00)$ & (1.06) & (1.09) & (1.12) & $(0.89)$ & $(0.97)$ & $(1.02)$ \\
\hline \multirow{2}{*}{ Nivel educativo } & 4.67 & 5.21 & 6.11 & 6.19 & 6.60 & 6.88 & 6.52 \\
\hline & $(2.40)$ & $(2.32)$ & $(2.04)$ & $(1.99)$ & $(2.18)$ & $(2.02)$ & $(2.09)$ \\
\hline
\end{tabular}

Notas. CCF-I= Consideración de las Consecuencias Inmediatas; CCF-F= Consideración de las Consecuencias Futuras; CCF-T= Consideración de las consecuencias por puntuación total.

En el ANOVA por grupo zonal se observan diferencias estadísticamente significativas en CCF-F $(F(6,803)=3,02, p=.006)$ y CCF-T $(F(6,803)=3.19, p=$ .004) y una tendencia de significación en CCF-I $(F(6,803)=1,83, p=.09)$. Los análisis post-hoc de Tukey (para las subescalas de CCF-F y CCF-T), revelan que no hay grupos homogéneos con asignación excluyente. El tamaño del efecto de la diferencia entre el conjunto de barrios más rico y el más pobre revela un efecto mediano de $g$ (Hedges) $=$ 0,42 para CCF-F, 0,44 para CCF-T y 0,27 para CCF-I. Asimismo, el tamaño del efecto de la diferencia entre el grupo 2 y el 7 en CCC-F es de $g=0,47$ y en CCF-I de $g=0,26$.

Debido a los efectos de la edad y nivel educativo sobre las puntuaciones de CCF, realizamos el mismo ANOVA anterior, con estas variables sociodemográficas como covariables. Encontramos que este efecto principal se reduce en todos los casos, pero 
mantiene tendencia a la significación en CCF-F, donde el efecto principal de los grupos zonales es de $F(6,808)=2,03, p=.06$, pero no en CCF-T, $F(6)=1,76, p=.13$

\section{Asociación de las puntuaciones de CCF con consumo de sustancias}

Para evaluar las asociaciones entre la CCF con el consumo de sustancias psicoactivas se estimaron los coeficientes de correlación entre las variables de estudio. A su vez, se realizan análisis de regresión lineal múltiple para evaluar si la CCF predice el consumo de tabaco, marihuana y bebidas alcohólicas. En la Tabla 5 se presentan los coeficientes de correlación entre la CCF, variables sociodemográficas (sexo, edad y nivel educativo) y consumo de sustancias psicoactivas. CCF-I correlaciona positivamente con la edad y consumo de tabaco; y negativamente con el nivel educativo. CCF-F se asocia positivamente con la edad, nivel educativo y negativamente con el consumo de marihuana y tabaco. Por último, CCF-T se asocia positivamente con el nivel educativo y negativamente con el consumo de marihuana y tabaco.

\section{Tabla 5}

Correlación entre las puntuaciones de la ECCF, variables sociodemográficas y frecuencia de consumo de sustancias psicoactivas.

\begin{tabular}{lccccccc}
\hline & 2 & 3 & 4 & 5 & 6 & 7 & $8 . \mathrm{CT}$ \\
\hline 1. CCF-I & $-.31^{* *}$ & $-.80^{* *}$ & $.13^{* *}$ & $-.25^{* *}$ & -.01 & .04 & $.07^{*}$ \\
2. CCF-F & & $.82^{* *}$ & $.06^{*}$ & $.14^{* *}$ & -.02 & $-.07^{*}$ & $-.09^{* *}$ \\
3. CCF-T & & & -.04 & $.24^{* *}$ & -.01 & $-.07^{*}$ & $-.10^{* *}$ \\
4. Edad & & & -.03 & $-.23^{* *}$ & $-.26^{* *}$ & $-.11^{* *}$ \\
5. NE & & & & $.15^{* *}$ & .03 & $-.09^{* *}$ \\
6. BA & & & & & $.30^{* *}$ & $.14^{* *}$ \\
7. CM & & & & & & $.20^{* *}$ \\
\hline
\end{tabular}

Notas. $\mathrm{CCF}-\mathrm{I}=$ Consideración de las Consecuencias Inmediatas; $\mathrm{CCF}-\mathrm{F}=$ Consideración de las Consecuencias Futuras; CCF-T= Consideración de las consecuencias por puntuación total; NE= Nivel educativo; $\mathrm{BA}=$ Bebidas alcohólicas; $\mathrm{CM}=$ Consumo de marihuana; $\mathrm{CT}=$ Consumo de tabaco.

En la Tabla 6 se presenta el resumen del análisis de regresión realizado para el consumo de tabaco, marihuana y bebidas alcohólicas. En el Paso 1 se controló por edad, sexo y nivel educativo de los participantes, debido a que son variables que provocan diferencias estadísticamente significativas entre grupos tanto en CCF como en consumo de sustancias. En el primer modelo, CCF-F es el único en predecir el consumo de tabaco, con una asociación negativa. En el segundo modelo, CCF-I es el único dominio en predecir el consumo de marihuana, con una asociación positiva. Por último, ni CCF-F ni CCF-I logran predecir el consumo de bebidas alcohólicas. 
Tabla 6

Resumen de los análisis de regresión lineal múltiple realizados

\begin{tabular}{|c|c|c|c|c|c|c|c|c|}
\hline \multicolumn{3}{|c|}{ Tabaco } & \multicolumn{3}{|c|}{ Marihuana } & \multicolumn{3}{|c|}{ BA } \\
\hline & B & $\mathrm{p}$ & & $\beta$ & $\mathrm{p}$ & & $\beta$ & $\mathrm{p}$ \\
\hline Paso 1 & & & Paso 1 & & & Paso 1 & & \\
\hline Edad & -.11 & .00 & Edad & -.26 & .00 & Edad & -.22 & .00 \\
\hline Sexo & .00 & .86 & Sexo & -.12 & .00 & Sexo & -.19 & .00 \\
\hline $\mathrm{NE}$ & -.09 & .00 & $\mathrm{NE}$ & .03 & .29 & $\mathrm{NE}$ & .15 & .00 \\
\hline Paso 2 & & & Paso 2 & & & & & \\
\hline Edad & -.11 & .00 & Edad & -.27 & .00 & & & \\
\hline Sexo & .00 & .87 & Sexo & -.12 & .00 & & & \\
\hline $\mathrm{NE}$ & -.08 & .00 & $\mathrm{NE}$ & .05 & .08 & & & \\
\hline CCF-F & -.07 & .01 & CCF-I & .08 & .00 & & & \\
\hline
\end{tabular}

Notas. $\mathrm{BA}=$ Bebidas alcohólicas; $\mathrm{NE}=$ Nivel educativo CCF-I $=$ Consideración de las Consecuencias Inmediatas; CCF-F= Consideración de las Consecuencias Futuras.

\section{Discusión}

Utilizando por primera vez una muestra representativa (en este caso de la población de Montevideo) este estudio tuvo por objetivo general avanzar en el conocimiento sobre el constructo consideración de las consecuencias futuras y la versión en español de la escala disponible para evaluarlo. Para ello, nos propusimos cuatro objetivos específicos que discutimos a continuación.

Nuestro primer objetivo fue analizar la estructura factorial y confiabilidad de la escala CCF en castellano en una muestra representativa. Nuestros resultados señalan, como estudios previos con muestras de conveniencia, tanto en español como otras lenguas (Joireman et al., 2012; McKay et al., 2016; Vásquez-Echeverría et al., 2018,), que la solución de dos factores correlacionados es la más adecuada y que ambos factores poseen adecuada confiabilidad, aunque algo reducida para CCF-F. Además, la calidad de las cargas es muy alta, con valores siempre cercanos o superiores a .40. Otras soluciones testeadas, concretamente la bifactor, presentan problemas en los índices de ajustes y/o saturaciones. Por lo tanto, la acumulación de evidencia, en este caso con una muestra muy diversa, sugiere que los investigadores deben considerar limitar el uso de la puntuación CCF-T y preferir la utilización de las puntuaciones de las subescalas CCF-I y CCF-F (Joireman \& King, 2016).

Nuestro segundo objetivo fue disponibilizar a la comunidad académica las puntuaciones percentiles de la referencia a la norma de Montevideo. Ofrecemos las puntuaciones para las subescalas y la CCF-T para toda la muestra y, en el caso de la CCFI, por sexo y grupo de edad, debido a las diferencias estadísticamente significativas halladas en dicha subescala. 
Respecto al tercer objetivo, analizar la distribución territorial de las puntuaciones en $\mathrm{CCF}$, encontramos evidencia mixta al respecto a que las personas de menor nivel socioeconómico presentan mayor orientación hacia el presente. Los resultados sugieren que los dos grupos zonales de menor educación y menor valor de vivienda presentan menores puntuaciones en todas las puntuaciones de CCF, especialmente en CCF-F. Esto replica lo ya fue reportado por Vásquez-Echeverría et al. (2019), en una muestra más reducida de padres de Montevideo. Sin embargo, el ordenamiento de las puntuaciones de CCF por grupo zonal no es estrictamente jerárquico ni consecutivo y no se encontraron diferencias significativas en los análisis post-hoc entre los grupos (incluido el de menor nivel socioeconómico). Esto puede deberse a que este efecto solo es notorio en agrupamientos donde existen contextos de pobreza marcados (como sucede en los grupos zonales 1 y 2) y luego de alcanzar un umbral de necesidades básicas satisfechas, la distribución de la consideración de las consecuencias futuras varía en función de otras características del entorno o de la personalidad. Por ejemplo, Pepper y Nettle (2017) señalan que la constelación comportamental de la privación (que incluye una mayor orientación temporal hacia el presente) se reproduce en función de incertidumbre a nivel laboral y el bajo control sobre el futuro en términos económicos, de educación y de salud, algo que en Uruguay es relativamente homogéneo entre los contextos de ingresos medios y medios-altos, pero no así en los contextos de pobreza. En estos últimos contextos es dónde se presentan mayores dificultades de acceso a la salud, mayores problemas de seguridad pública y mayor tasa de deserción de la educación obligatoria. En esta línea, nuestros hallazgos señalan que el nivel educativo explica parte de estas diferencias en orientación temporal, pero es necesario explorar otras características para entender las diferencias entre los barrios de menores ingresos en su CCF. De todas formas, nuestros resultados reafirman la idea de que la distribución territorial de la orientación temporal no es aleatoria y amerita futuras investigaciones al respecto.

Por último, nuestro cuarto objetivo fue explorar la asociación de las puntuaciones de la ECCF en relación a la frecuencia de consumo de sustancias psicoactivas. Si bien son efectos pequeños, encontramos evidencia que ambas subescalas explican el consumo de ciertas sustancias, controlando por variables sociodemográficas. Por un lado, las personas que se orientan a las consecuencias inmediatas tienden a consumir más marihuana. La marihuana es una sustancia que provoca placer hedónico inmediato y reduce la posibilidad de realizar otras actividades mientras se consume, por lo que una mayor orientación hacia la búsqueda de efectos inmediatos (mayor CCF-I) puede 
favorecer su consumo. En cambio, el tabaco produce efectos perjudiciales a largo plazo y tiene beneficios hedónicos más reducidos. Por lo que una mayor CCF-F, puede hacer que las personas consideren las ventajas a largo plazo de la reducción del consumo (e.g.: reducir las probabilidades de desarrollar un cáncer de pulmón en 20 años).

Si bien este estudio empleó una muestra representativa, no está exento de limitaciones. En primer lugar, la muestra es representativa a nivel departamental (regional) y no nacional, por lo que los resultados deben ser usados con cautela si se quieren extrapolar a todo el Uruguay u otros países hispanohablantes, en especial las puntuaciones de la referencia a la norma. Segundo, en lo que hace a los resultados territoriales, futuros estudios, tomando los indicios que aquí se aportan, podrían elaborar diseños multinivel, que serían más apropiados para indagar a fondo el impacto de los agrupamientos de segundo orden en el comportamiento individual. Para esto, algunos de nuestros análisis se podrían complementar con datos de criminalidad, desempleo, disponibilidad de centros de salud en cada barrio o grupo zonal para indagar si el menor control ambiental explica la variabilidad en la consideración de las consecuencias futuras a nivel zonal. Por último, el consumo de sustancias fue autoreportado, lo cual puede inducir sesgos en las respuestas por deseabilidad social. Futuros estudios pueden beneficiarse de estos hallazgos a nivel poblacional para ampliar nuestros resultados de investigación y profundizar sobre la relación de la CCF con variables sociodemográficas de las comunidades y el consumo de sustancias psicoactivas. 


\section{Referencias}

Adams, J. (2012). Consideration of immediate and future consequences, smoking status, and body mass index. Health Psychology, 31(2), 260-263. https://doi.org/10.1037/a0025790

Adams, J., \& White, M. (2009). Time perspective in socioeconomic inequalities in smoking and body mass index. Health Psychology, 28(1), 83-90. https://doi.org/10.1037/0278-6133.28.1.83

Alvarez-Nuñez, L. (2018). Confiabilidad y validez de la Escala Consideración de las Consecuencias Futuras en español Cómo pensamos en las consecuencias inmediatas (pero no las distantes) de nuestras acciones se asocia a un patrón conductual saludable. Tesis de maestría no publicada. Universidad de la República, Uruguay. https://www.colibri.udelar.edu.uy/jspui/bitstream/20.500.12008/20038/1/Alvarez\%20 Nu\%C3\%B1ez\%2C\%20Luc\%C3\%ADa.pdf

Balliet, D., \& Ferris, D. L. (2013). Ostracism and prosocial behavior: A social dilemma perspective. Organizational Behavior and Human Decision Processes, 120(2), 298-308. https://doi.org/10.1016/j.obhdp.2012.04.004

Beenstock, J., Adams, J., \& White, M. (2011). The association between time perspective and alcohol consumption in university students: cross-sectional study. The European Journal of Public Health, 21(4), 438-443. https://doi.org/10.1093/eurpub/ckp225

Byrne, B. (2013). Testing Change Over Time The Latent Growth Curve Model. In Structural equation modeling with Mplus: Basic concepts, applications, and programming (pp. 313-344). New York: Routledge.

Camus, G., Berjot, S., \& Ernst-Vintila, A. (2014). Validation française de l'échelle de prise en considération des conséquences futures de nos actes (CFC-14). Revue Internationale de Psychologie Sociale, 27(1), 35-63.

Cicchetti, D. V. (1994). Guidelines, criteria, and rules of thumb for evaluating normed and standardized assessment instruments in psychology. Psychological Assessment, 6(4), 284-290. https://doi.org/10.1037/1040-3590.6.4.284

Daugherty, J. R., \& Brase, G. L. (2010). Taking time to be healthy: Predicting health behaviors with delay discounting and time perspective. Personality and Individual Differences, pp. 202-207. https://doi.org/10.1016/j.paid.2009.10.007

Haushofer, J., \& Fehr, E. (2014). On the psychology of poverty. Science, 344(6186), 862867. https://doi.org/10.1126/science.1232491

Instituto Nacional de Estadística. (2011). Resultados del Censo de Población 2011: población, crecimiento y estructura por sexo y edad.

Joireman, J., Balliet, D., Sprott, D., Spangenberg, E., \& Schultz, J. (2008). Consideration of future consequences, ego-depletion, and self-control: Support for distinguishing between CFC-Immediate and CFC-Future sub-scales. Personality and Individual Differences, 45(1), 15-21. https://doi.org/10.1016/j.paid.2008.02.011

Joireman, J., Becker, C., Barbosa-Leiker, C., \& Duell, B. (2005). Aggression as a function of concern with future consequences and anticipated interaction with an aggressive peer. Poster presented at the $6^{\text {th }}$ Annual Convention of the Society of Personality and Social Psychology Conference, New Orleans, LA. January 2005.

Joireman, J., \& King, S. (2016). Individual Differences in the Consideration of Future and (More) Immediate Consequences: A Review and Directions for Future 
Research. Social and Personality Psychology Compass, 10(5), 313-326. https://doi.org/10.1111/spc3.12252

Joireman, J., Shaffer, M. J., Balliet, D., \& Strathman, A. (2012). Promotion Orientation Explains Why Future-Oriented People Exercise and Eat Healthy: Evidence From the Two-Factor Consideration of Future Consequences-14 Scale. Personality and Social Psychology Bulletin, 38(10), 1272-1287. https://doi.org/10.1177/0146167212449362

Joireman, J., Strathman, A., \& Balliet, D. (2006). Considering Future Consequences An Integrative Model. In E. Sanna, J. Lawrence, \& E. Chang (Eds.), Judgments Over Time The Interplay of Thoughts, Feelings, and Behaviors (pp. 82-99). https://doi.org/10.1093/acprof:oso/9780195177664.003.0006

Lindsay, J. J., \& Strathman, A. (1997). Predictors of Recycling Behavior: An Application of a Modified Health Belief Model. Journal of Applied Social Psychology, 27(20), 1799-1823. https://doi.org/10.1111/j.1559-1816.1997.tb01626.x

McKay, M. T., Cole, J. C., \& Percy, A. (2015). Further evidence for a bifactor solution for the Consideration of Future Consequences Scale: Measurement and conceptual implications. Personality and Individual Differences, 83, 219-222. https://doi.org/10.1016/j.paid.2015.04.022

McKay, M. T., Morgan, G. B., van Exel, N. J., \& Worrell, F. C. (2015). Back to "the Future": Evidence of a Bifactor Solution for Scores on the Consideration of Future Consequences Scale. Journal of Personality Assessment, 97(4), 395-402. https://doi.org/10.1080/00223891.2014.999338

McKay, M. T., Perry, J. L., Percy, A., \& Cole, J. C. (2016). Evidence for the reliability and validity, but not the practical utility of the two-factor Consideration of Future Consequences Scale-14. Personality and Individual Differences, 98, 133-136. https://doi.org/10.1016/j.paid.2016.03.097

Murphy, L., Cadogan, E., \& Dockray, S. (2019). The Consideration of Future Consequences: Evidence for Domain Specificity Across Five Life Domains. Personality and Social Psychology Bulletin, 014616721987347. https://doi.org/10.1177/0146167219873478

Nigro, G., Cosenza, M., Ciccarelli, M., \& Joireman, J. (2016). An Italian translation and validation of the Consideration of Future Consequences-14 Scale. Personality and Individual Differences, 101, 333-340. https://doi.org/10.1016/j.paid.2016.06.014

Pepper, G. V., \& Nettle, D. (2017). The behavioural constellation of deprivation: Causes and consequences. Behavioral and Brain Sciences, 40(July), e314. https://doi.org/10.1017/S0140525X1600234X

Percy, A., McKay, M. T., Perry, J. L., \& Cole, J. C. (2018). Refining the clinical application of the consideration of future consequences scale -14. Current Psychology. https://doi.org/10.1007/s12144-018-9819-5

Ponce, J., \& Tubbio, M. (2013). Precios de inmuebles: aproximaciones metodológicas y aplicación empírica. Documento de Trabajo Del Banco Central Del Uruguay.

Strathman, A., Gleicher, F., Boninger, D. S., \& Edwards, C. S. (1994). The consideration of future consequences: Weighing immediate and distant outcomes of behavior. Journal of Personality and Social Psychology, 66(4), 742-752. https://doi.org/10.1037/0022-3514.66.4.742

Toepoel, V. (2010). Is consideration of future consequences a changeable construct? Personality and Individual Differences, 48(8), 951-956. https://doi.org/10.1016/j.paid.2010.02.029

Vásquez-Echeverría, A., Antino, M., Alvarez-Nuñez, L., \& Rodríguez-Muñoz, A. (2018). Evidence for the reliability and factor solution of the CFCS-14 in Spanish: A 
multi-method validation in Spain and Uruguay. Personality and Individual Differences, 123, 171-175. https://doi.org/10.1016/j.paid.2017.11.021

Vásquez-Echeverría, A., Tomás, C., \& Cruz, O. (2019). The development of episodic foresight in preschoolers: the role of socioeconomic status, parental future orientation, and family context. Psicologia: Reflexão e Crítica, 32(1), 12. https://doi.org/10.1186/s41155-019-0125-4

Vásquez Echeverría, A., Martín, A., Ortuño, V., Esteves, C., \& Joireman, J. (2017). Adaptación y validación inicial al castellano de la Escala Consideración de las Consecuencias Futuras. Revista Iberoamericana de Diagnóstico y Evaluación-e Avaliação Psicológica, 44(2), 5-15.

Viladrich, C., Angulo-Brunet, A., \& Doval, E. (2017). Un viaje alrededor de alfa y omega para estimar la fiabilidad de consistencia interna. Anales de Psicología, 33(3), 755. https://doi.org/10.6018/analesps.33.3.268401

Zhang, Z., Kong, M., Zhang, L., \& Li, Z. (2015). Consideration of future consequences: Preliminary evidences for a four-factor distinction. Personality and Individual Differences, 87, 99-104. https://doi.org/10.1016/j.paid.2015.07.022

Received: $2016 / 07 / 29$

Accepted: 2020/03/30 


\begin{tabular}{|c|c|c|c|c|c|c|c|c|c|}
\hline $\begin{array}{l}\text { Anexo } 1 \\
\text { Baremos }\end{array}$ & $\operatorname{ra~Mc}$ & tevide & o de la 1 & scala de & Conside & ación de & is Consect & ncias Fut & \\
\hline & & & & & & & CF-I & & \\
\hline & CCF- & CCF- & & $\mathrm{Se}$ & & & Rang & etario & \\
\hline Percentil & $\mathrm{F}$ & $\mathrm{T}$ & CCF-I & Hombre & Mujer & A. Joven & A. medio & A. tardía & A. mayor \\
\hline 5 & 2.43 & & 1.00 & 1.00 & 1.00 & 1.00 & 1.00 & 1.00 & 1.00 \\
\hline 10 & 2.86 & & 1.33 & 1.50 & 1.33 & 1.33 & 1.33 & 1.50 & 1.50 \\
\hline 25 & 3.71 & & 2.00 & 2.17 & 2.00 & 2.00 & 2.00 & 2.29 & 2.50 \\
\hline 33.3 & 4.14 & & 2.33 & 2.50 & 2.17 & 2.17 & 2.33 & 2.66 & 2.83 \\
\hline 50 & 4.57 & & 3.00 & 3.17 & 3.00 & 2.83 & 3.00 & 3.17 & 3.83 \\
\hline 66.6 & 5.14 & 5.15 & 3.67 & 3.67 & 3.50 & 3.50 & 3.50 & 3.50 & 4.32 \\
\hline 75 & 5.29 & & 4.00 & 4.17 & 4.00 & 4.00 & 4.00 & 4.17 & 5.00 \\
\hline 90 & 6.14 & & 5.00 & 5.00 & 5.00 & 4.83 & 5.00 & 5.00 & 5.50 \\
\hline 95 & 6.43 & 6.38 & 5.67 & 5.83 & 5.61 & 5.50 & 5.78 & 6.00 & 6.24 \\
\hline
\end{tabular}

Notas. CCF-I= Consideración de las Consecuencias Inmediatas; $\mathrm{CCF}-\mathrm{F}=$ Consideración de las

Consecuencias Futuras; CCF-T= Consideración de las consecuencias por puntuación total; A= Adulto. 